\title{
An Improved PSO Algorithm and Its Application on Fault Diagnosis
}

\author{
X.L. Liu, L.H. Cao, S.T. Wang, J.N. Li,Y. Huang \\ Chongqing communication Institute \\ Chongqing, China
}

\begin{abstract}
For the disadvantages of PSO (Particle Swarm Optimization) algorithm, such as premature convergence and easily getting into local extremum, an improved PSO algorithm was presented in this paper. On the one hand, the population with worse performance moved near the global optimization value of the other population with certain probability; on the other hand, one population was randomly chosen to mutate to stimulate the particles jump out the local extremum when the two populations continuously trapped into the same local extremum. The simulation results showed that the improved PSO had a better optimization performance. SVM (Support Vector Machine) trained the improved PSO was applied to fault diagnosis of diesel engine valve. The simulation results showed that the improved PSO-SVM acquired higher accuracy.
\end{abstract}

Keywords-particle swarm optimization; multi-population; support vector machine; fault diagnosis

\section{INTRODUCTION}

PSO(Particle Swarm Optimization) is a kind of Swarm Optimization algorithm put forward by Eberhart and Kennedy in 1995. As it has the characteristics of simple realization, strong currency and good optimization performance, it is applied on many fields at present, such as multi-objective optimization, fault diagnosis, intelligent control and so on[1-4]. But the basic PSO algorithm has disadvantages of prematurity and easily trapping into local extremum. For these shortcomings of basic PSO algorithm, an improved PSO was introduced in this paper, which used two populations to coevolve to improve the local search ability.

Fault diagnosis can be viewed as pattern classification problems, and SVM which is a kind of machine learn algorithms based on Statistical Learning Theory has the adaptive generation ability[5-6]. However, the SVM performance is largely related to parameters of the model, so the improved PSO was applied to optimize parameters of SVM to enhance the classification ability in the paper. The simulation results showed that the method proposed in the paper had a better diagnosis results.

\section{IMPROVED PSO ALGORITHM WITH TWO POPULATIONS}

\section{A. The Principle of Basic PSO Algorithm}

In basic PSO algorithm, suppose search space is $D$ dimension, size of population is $m$. The position and velocity of $i$-th particle are represented as $X_{i}=\left[x_{i 1}, x_{i 2}, \cdots x_{i D}\right]$ and $V_{i}=\left[v_{i 1}, v_{i 2}, \cdots v_{i D}\right]$ respectively, $i=1,2 \cdots, m$, the variation of search space is $\left[X_{\min }, X_{\max }\right]$. Particle's position $X_{i}$ is

\author{
Y.P. Li \\ PLA77556 troop \\ Tibet, China
}

substituted in object function to calculate fitness value $f(i)$. Then by evaluating $f(i)$, the individual extremum $P_{i}=\left[p_{i 1}, p_{i 2}, \cdots p_{i D}\right] \quad$ and global extremum $P_{g}=\left[p_{g 1}, p_{g 2}, \cdots p_{g D}\right]$ are obtained. All particles' velocities and positions can be updated according to equations (1) and (2):

$$
\begin{gathered}
V_{i}(t+1)=w V_{i}(t)+c_{1}\left[P_{i}(t)-X_{i}(t)\right]+c_{2}\left[P_{g}(t)-X_{i}(t)\right](1) \\
X_{i}(t+1)=X_{i}(t)+V_{i}(t)(2)
\end{gathered}
$$

where $w$ is inertia weight, $c_{1}$ and $c_{2}$ are positive acceleration constants.

\section{B. The Principle and Flow of Improved PSO Based on Two Populations Co-evolution}

In the improved algorithm, on the one hand, the particles of worse population move near the global extremum of the other population with certain probability. On the other hand, one population was randomly chosen to mutate to stimulate the particles jump out the local extremum when the two populations continuously trapped into the same local extremum. The diversity of population was enhanced and the local search ability was improved by this way.

The flow of improved PSO algorithm is as follows:

(1) Initialization of populations, suppose the number of dimensions of optimal problem is $D$, the size of population is $m$, the maximal iterations is $K$, the range of search space is $\left[x_{\min }, x_{\max }\right] \quad, \quad$ inertial coefficient $w(k+1)=0.9-(k / K) * 0.5$,set $c_{1}, c_{2}$; Initialization the particles' position $X_{1}, X_{2}$ and velocity $V_{1}, V_{2}$ randomly, which are all matrix of $m^{*} D$.

(2) Making current positions $X_{1}$ and $X_{2}$ are individual best positions $P_{1}$ and $P_{2}$, current individual fitnesses are individual extrema $F_{1}$ and $F_{2}$. Through evaluation, obtaining two populations' global best positions $p_{g}^{1}, p_{g}^{2}$ and global extrema $f_{\text {best }}^{1}, f_{\text {best }}^{2}$ separately; $k=0$;

(3) Updating the two populations' positions according to 
formulas (1) and (2);

(4) Calculating every particle’s fitness; Obtaining personal best positions $p_{1}, p_{2}$ and individual extrema $f_{1}, f_{2}$ by evaluating every particle; Obtaining global best positions $p_{g}^{1}$, $p_{g}^{2}$ and global extrema $f_{\text {best }}^{1}, f_{\text {best }}^{2}$ by evaluating all particles; Obtaining the optimization value $f_{\text {best }}$ by evaluating $f_{\text {best }}^{1}$ and $f_{\text {best }}^{2}$.

(5) If $f_{\text {best }}^{1}<f_{\text {best }}^{2}$, updating $p_{g}^{2}$ according to formula (3), vice versa.

$$
p_{g}^{2}=\left\{\begin{array}{ccc}
p_{g}^{1}+R *\left(p_{g}^{2}-p_{g}^{1}\right) & \text { if } & \operatorname{rand}(0,1)<\exp \left(f_{1}-f_{2}\right) \\
p_{g}^{2} & \text { else }
\end{array}\right.
$$

where $R$ is random numbers valued between 0 and 1 .

(6) If $f_{\text {best }}^{1}=f_{\text {best }}^{2}$, a Cauchy mutation operator is added to $P_{g}^{1}$ to generate a random position far away from $P_{g}^{1}$, as show in formula (4).

$$
p_{g}^{1}=p_{g}^{1}+\frac{\operatorname{rand}(0,1) \cdot \theta}{\pi \cdot\left(\left(p_{g}^{1}\right)^{2}+\theta^{2}\right)}
$$

where $\theta$ is a pre-set parameter.

(7) Judging the algorithm whether to meet termination conditions, if it does, the algorithm is over, and output the global extremum as a final result; conversely goes back to step (3).

\section{FAUlT DiAgNOSIS BASED ON SVM OPTIMIZED BY IMPROVED PSO}

\section{A. SVM Optimized by Improved PSO}

When SVM is applied to pattern recognition, firstly, the input vectors from nonlinear separable space are mapped to characteristic space; then the optimal separation hyperplane is generated to carry out pattern classification in this characteristic space.

$$
\Phi(w, \xi)=\frac{1}{2} w^{\mathrm{T}} w+C \sum_{i=1}^{s} \xi_{i}
$$

Constraint condition is:

$$
y_{i}\left(w^{\mathrm{T}} \phi\left(x_{i}\right)+b\right) \geq 1-\xi_{i}, i=1,2, s
$$

where, $w$ and $b$ are weights and bias of the optimal hyberplane separately; $\xi$ are slack variables which are introduced to solve nonlinear classification problems, $\xi_{i} \geq 0$; $C$ is penalty factor; $\varphi(x)$ is the kernel function which allows input vectors to be mapped to characteristic space; $s$ is sample number. This constrained optimal problem can be solved by Lagrange multiplier method:

$$
J(w, b, \alpha)=\frac{1}{2} w^{\mathrm{T}} w+C \sum_{i=1}^{s} \xi_{i}+\sum_{i=1}^{s} \alpha_{i}\left[y_{i}\left(w^{\mathrm{T}} \phi\left(x_{i}\right)+b\right)-\left(1-\xi_{i}\right)\right]
$$

where $\alpha_{i}$ is Lagrange multiplier, the two optimal conditions are obtained by calculating derivative of function $J w$ and $b$ equal to 0 .

$$
\left\{\begin{array}{l}
\frac{\partial J}{\partial w}=0 \\
\frac{\partial J}{\partial b}=0
\end{array}\right.
$$

The dual of above problem can be established according to (7) and (8):

Given training samples $\left(x_{i}, y_{i}\right)$, the optimal objective function is:

$$
Q(\alpha)=\frac{1}{2} \sum_{i=1}^{s} \sum_{j=1}^{s} \alpha_{i} \alpha_{j} y_{i} y_{j} K\left(x_{i}, x_{j}\right)-\sum_{i=1}^{s} \alpha_{i}
$$

Constraint condition is:

$$
\left\{\begin{array}{l}
\sum_{i=1}^{s} \alpha_{i} y_{i}=0 \\
0 \leq \alpha_{i} \leq C
\end{array}\right.
$$

where, $K\left(x_{i}, x_{j}\right)=\phi\left(x_{i}\right)^{\mathrm{T}} \cdot \phi\left(x_{j}\right), K\left(x_{i}, x_{j}\right)$ is called kernel function whose existence is guaranteed by Mercer theorem. Common kernel functions are polynomial, RBF and sigmoid. Optimal weight vectors $w$ and bias $b$ can be solved and then optimal hyperplane $\left(w^{T} \cdot \varphi(x)+b=0\right)$ is ascertained after determining Lagrange multiplier $\alpha_{i}$.

RBF kernel function is chose in the paper, that is:

$$
\phi(x)=\exp \left(-\frac{1}{2 \sigma^{2}}\left\|x-x_{i}\right\|^{2}\right)
$$

According to above discussion, SVM performance is mainly influenced by penalty factor $C$ and parameter $\sigma$ in kernel function. So in order to find the optimal $C$ and $\sigma$, improved PSO algorithm is introduced to train SVM in the paper.

When the improved PSO algorithm is used to optimize parameters of SVM, the particles' positions are encoded as 
$[c, \sigma]$, the fitness function is defined as $f=\frac{1}{1+a}$, where $a$

is classification accuracy rate, $f$ is varied from 0.5 and 1 . Finally the global best position found by the improved PSO algorithm contains the optimization parameters of RBF NN, such as $C, \sigma$.

The flow of SVM trained by the improved PSO is as show in Figure 1.

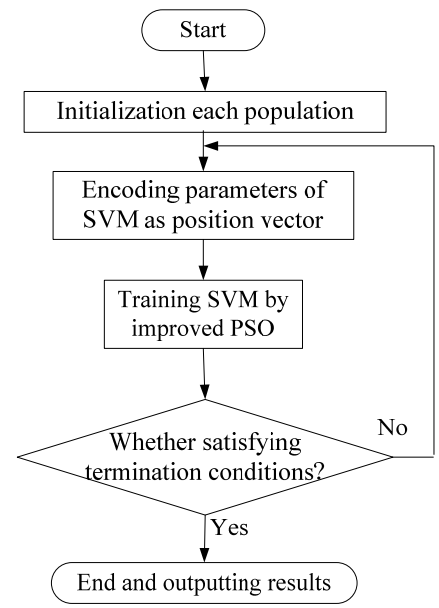

FIGURE I. THE FLOW OF SVM OPTIMIZED BY IMPROVED PSO.

\section{B. Fault Diagnosis Example}

The paper used experimental data as shown in Table 1 in which the fault symptoms are wavelet packets energy spectra in nodes of the third layer of cylinder head vibration signal and codes for fault types from 0 to 4 stands for normal state, small exhaust valve clearance, big exhaust valve clearance, mild leakage and serious leakage respectively. The experiment was carried out on 6135D diesel engine without load, and the sampling frequency was $25 \mathrm{KHz}$. In the experiment, including normal state, a total of 5 types of vibration signals were measured by using acceleration sensor installed in cylinder head, and 9 sets of data were measured at each state, so there were 45 fault samples in all.

TABLE I. FaUlt Diagnosis SAMples (PARTial DATA).

\begin{tabular}{|c|c|c|c|c|c|c|c|c|c|}
\hline $\begin{array}{c}\text { Symptoms } \\
\text { Samples No }\end{array}$ & $\mathbf{1}$ & $\mathbf{2}$ & $\mathbf{3}$ & $\mathbf{4}$ & $\mathbf{5}$ & $\mathbf{6}$ & $\mathbf{7}$ & $\mathbf{8}$ & $\begin{array}{c}\text { Fault } \\
\text { types }\end{array}$ \\
\hline $\mathbf{1}$ & 0.0344 & 0.0512 & 0.0955 & 0.1076 & 0.2443 & 0.2869 & 0.0929 & 0.0872 & 0 \\
\hline $\mathbf{2}$ & 0.0331 & 0.0433 & 0.4135 & 0.0949 & 0.0175 & 0.0113 & 0.3412 & 0.0451 & 1 \\
\hline $\mathbf{3}$ & 0.0335 & 0.0496 & 0.1506 & 0.0773 & 0.0735 & 0.1647 & 0.1502 & 0.3006 & 2 \\
\hline $\mathbf{4}$ & 0.0369 & 0.0372 & 0.1169 & 0.0551 & 0.0242 & 0.0624 & 0.5064 & 0.1610 & 3 \\
\hline $\mathbf{5}$ & 0.0454 & 0.0858 & 0.1665 & 0.1005 & 0.0379 & 0.0859 & 0.3302 & 0.1478 & 4 \\
\hline $\mathbf{6}$ & 0.0503 & 0.0461 & 0.1437 & 0.0911 & 0.2193 & 0.2559 & 0.0959 & 0.0979 & 0 \\
\hline $\mathbf{7}$ & 0.0363 & 0.0337 & 0.3118 & 0.0633 & 0.0103 & 0.0179 & 0.4837 & 0.0430 & 1 \\
\hline $\mathbf{8}$ & 0.0510 & 0.0503 & 0.1011 & 0.0989 & 0.0890 & 0.1395 & 0.2111 & 0.2592 & 2 \\
\hline $\mathbf{9}$ & 0.0332 & 0.0414 & 0.3296 & 0.1256 & 0.0360 & 0.0520 & 0.2795 & 0.1027 & 3 \\
\hline $\mathbf{1 0}$ & 0.0483 & 0.0809 & 0.2060 & 0.1070 & 0.0269 & 0.0654 & 0.2431 & 0.2224 & 4 \\
\hline
\end{tabular}

When improved PSO-SVM is applied to fault diagnosis, the fitness function $f(i)$ (namely performance function of network) is defined as follows:

$$
f(i)=1 / 1+a
$$

where $a$ is the classification accuracy.

$2 / 3$ of samples at each state were chosen as training samples, the others as test samples. The parameters needed in PSO-SVM were defined as follows: : $w=1-0.3 \cdot \mathrm{k} / \mathrm{K}$; $c_{1}=2 ; c_{2}=2 m=100 ; K=2000 ; E_{-}$goal $=1 * 10^{-3}$; and in SVM, the parameters were defined as follows: $C=10$; $\sigma=1$; The algorithm's stopping condition were reaching max iterations $K$ or meeting training error goal $E_{-}$goal . The classification accuracy was shown in Talble 2.

TABLE II. RESUlts of FAUlt Diagnosis By DifFERENT AlgORITHMS.

\begin{tabular}{c|c}
\hline Different algorithms & Accuracy \\
\hline IPSO-SVM & $96.7 \%$ \\
\hline PSO-SVM & $93.3 \%$ \\
\hline SVM & $86.7 \%$ \\
\hline
\end{tabular}

From Table 2 it can be seen that the improved PSO-SVM algorithm's diagnosis accuracy was the best, so the optimization algorithm proposed in this paper improved classification performance of SVM.

\section{CONCLUSIONS}

The key to solve problems of premature and trapping into local extremum in PSO is to keep the population's diversity and to avoid all particles tending to the same local extremum. Therefore, the improved PSO algorithm with two populations was proposed in this paper, and the advantages of this algorithm is that populations not only make full use of its own information, but also utilize optimal information of the other population. Cauchy mutation operator is added to one population's best position when the two populations find out the same optimal value. Then the improved PSO algorithm is applied to train SVM, and the simulation results showed it could enhance the classification ability of SVM and improve the accuracy of fault diagnosis, so the improved PSO-SVM model proposed in this paper will start a new way for fault diagnosis.

\section{ACKNOWLEDGEMENTS}

This work is supported by Chongqing college and universities good science and research translation significant project (No: KJZH14112).

\section{REFERENCES}

[1] Gonggui Chen, Lilan Liu, Peizhu Song, Yangwei Du. Chaotic improved PSO-based multi-objective optimization for minimization of power losses and $\mathrm{L}$ index in power systems[J]. Energy Conversion and Management, 86: 548-560, 2014.

[2] F. Khoshahval, A. Zolfaghari, H. Minuchehr, M.R. Abbasi. A new hybrid method for multi-objective fuel management optimization using parallel PSO-SA[J]. Progress in Nuclear Energy, 76: 112-121, 2014.

[3] Xiao X, Dow E R, Eberhart R, et al. A Hybrid self-organizing maps and particle swarm optimization approach[J]. Concurrency and Computation-Particle \& Experience,16(9):895-915, 2004.

[4] Rabindra Kumar Sahu, Sidhartha Panda, G.T. Chandra Sekhar. A novel hybrid PSO-PS optimized fuzzy PI controller for AGC in multi area 
interconnected power systems[J]. Electrical Power and Energy Systems,

(64): 880-893, 2015.

[5] Cortes C, Vapnic V. Support Vector networks[J]. Machine Learning, 20(1) 1-25, 1995.

[6] V. Muralidharan, V. Sugumaran, V. Indira. Fault diagnosis of monoblock centrifugal pump using SVM[J]. Engineering Science and Technology, an International Journal,17: 152-157, 2014. 